\title{
oints
}

(1)

\section{Recurrent anterior shoulder dislocation with glenoid bone loss and concurrent irreparable rotator cuff tear in a young patient: case report}

\author{
DARIO PETRICCIOLI, CELESTE BERTONE, GIACOMO MARCHI \\ Department of Orthopaedics, Clinical Institute "Città di Brescia", Italy
}

\begin{abstract}
Full-thickness rotator cuff tear associated with glenohumeral anterior dislocation is a rare injury in patients younger than 40 years of age. We here report a case of recurrent anterior shoulder dislocation with glenoid bone loss and concurrent irreparable rotator cuff tear in a young patient. This is, to the best of our knowledge, the first such case reported in the literature. We also discuss the type of surgical treatment adopted and the measures that can be undertaken to address both instability and massive rotator cuff tear.
\end{abstract}

Key Words: massive rotator cuff tear, shoulder dislocation, latissimus dorsi transfer.

\section{Introduction}

Full-thickness rotator cuff tear associated with glenohumeral anterior dislocation is common in patients older than 40 years of age, possibly due to weakening of posterior structures. Younger patients, with stronger posterior shoulder structures, are less prone to suffer a rotator cuff tear when a shoulder dislocation is sustained. To date the literature offers little guidance on the management of this combination of pathologies. In cases of recurrent dislocation associated with rotator

\footnotetext{
Corresponding Author:

Celeste Bertone, MD

Department of Orthopaedics, Clinical Institute

"Città di Brescia"

Via Gualla 15, 25123 Brescia, Italy

Phone: +39 3407078408

Fax: +39030 3710351

E-mail: celeste.bertone@gmail.com
}

cuff tear, treatment of instability should be proposed, whereas concomitant repair of the cuff depends on a series of factors determining the feasibility of performing the procedure: tear size, the presence of fatty infiltration, and the patient's age and level of motivation. We here present the case of a young man with recurrent anterior glenohumeral instability and concomitant irreparable posterosuperior rotator cuff tear.

\section{Case report}

A 31-year-old, right-handed male manual worker presented at our clinic with a long history of right-sided recurrent anterior shoulder dislocation and pain with severe function limitation in overhead activities. A general clinical examination was performed and showed the patient's general conditions to be good. His right shoulder active range of motion (ROM) was significantly decreased, particularly in forward flexion (100 degrees); his passive ROM was normal. Muscle testing revealed severe weakness of the supraspinatus and infraspinatus, which were very painful on strength testing; the Jobe and Whipple tests, used to evaluate the supraspinatus, and the external rotation lag sign (ERLS), used to evaluate the infraspinatus, were positive (the ERLS showed $75^{\circ}$ of automatic recall). Liftoff and belly-press tests were negative. There was no positive sulcus sign. The apprehension sign was painful at 60,90 and $120^{\circ}$ in the right shoulder, and negative on the left side. The fulcrum, relocation and push and pull tests were also positive. The patient's pre-operative Oxford Instability score was 7/48. His Western Ontario Shoulder Instability Index (WOSI) scores, measuring shoulder pain and disability, were as follows: physical symptoms 525 (52.5\%), sport/ recrea- 
tion/work 303 (75.5\%), lifestyle 234 (58.5\%), emotion $251(83.7 \%)$. His WOSI total score was 1313 (62.5\%), where 0 corresponds to full normal shoulder function. Neurovascular examination revealed no deficits.

A preliminary axial X-ray (Bernageau profile view) showed antero-inferior glenoid bone loss that was confirmed by a computed tomography scan. Magnetic resonance imaging was performed to evaluate the patient's rotator cuff: a complete posterosuperior rotator cuff tear with a stage 4 fatty degeneration was observed.

An arthroscopically-assisted latissimus dorsi (LD) transfer with an anterior glenoid bone graft was performed $(1,2)$. The patient was placed in the beach chair position. Complete detachment of the anteroinferior labrum with an enlarged capsule, a positive drive-through sign, and a reduction of the anteroposterior glenoid diameter were observed arthroscopically. The posterosuperior rotator cuff was torn and retracted and a humeral engaging Hill-Sachs defect was detected.

For the LD tendon harvesting, a skin incision about 5 to $6 \mathrm{~cm}$ long was made in the axillary crease orthogonal to the longitudinal axis of the upper arm, after which a structural tricortical graft was harvested from the middle section of the iliac crest in such a way as to obtain a slightly curved surface that exactly fitted the area of the glenoid defect. The bone graft was about $8 \mathrm{~mm}$ thick, 1 $\mathrm{cm}$ deep and about $3 \mathrm{~cm}$ wide. The graft was placed, under arthroscopic control, below the equator and level with the edge of the anterior glenoid with the inner cortical concave table oriented anteriorly. The graft was fixed with two cannulated self-tapping screws using a dedicated guide. After debridement of the subacromialsubdeltoid bursa and the greater tuberosity, a tenotomy of the long head of the biceps tendon was carried out. The LD tendon was then transferred arthroscopically through a tunnel created deep to the deltoid and posterior to the teres minor, passing in front of the triceps and the teres major. Postoperatively, the patient did well with no complications.

By the first follow-up examination there had been an obvious reduction in the level of shoulder pain. The patient's ROM continued to improve without apprehension but his shoulder girdle muscles showed noticeable atrophy. At 12 weeks, the patient progressed from passive to active physical therapy exercises. At six months post-surgery, the patient had regained a full ROM, except for a mild decrease in external rotation and some residual weakness without pain. The rehabilitation continued over the following three months with an aggressive program for LD strengthening. At one year post-surgery, the patient showed no instability, a full ROM, and no pain. Radiographic examinations showed good healing of the bone block and adequate preservation of the acromion-humeral interval (Fig. 1). Muscle strength was measured using a Biodex isokinetic dynamometer. We tested the patient with glenohumeral eccentric exercise in internal rotation (IR) and external
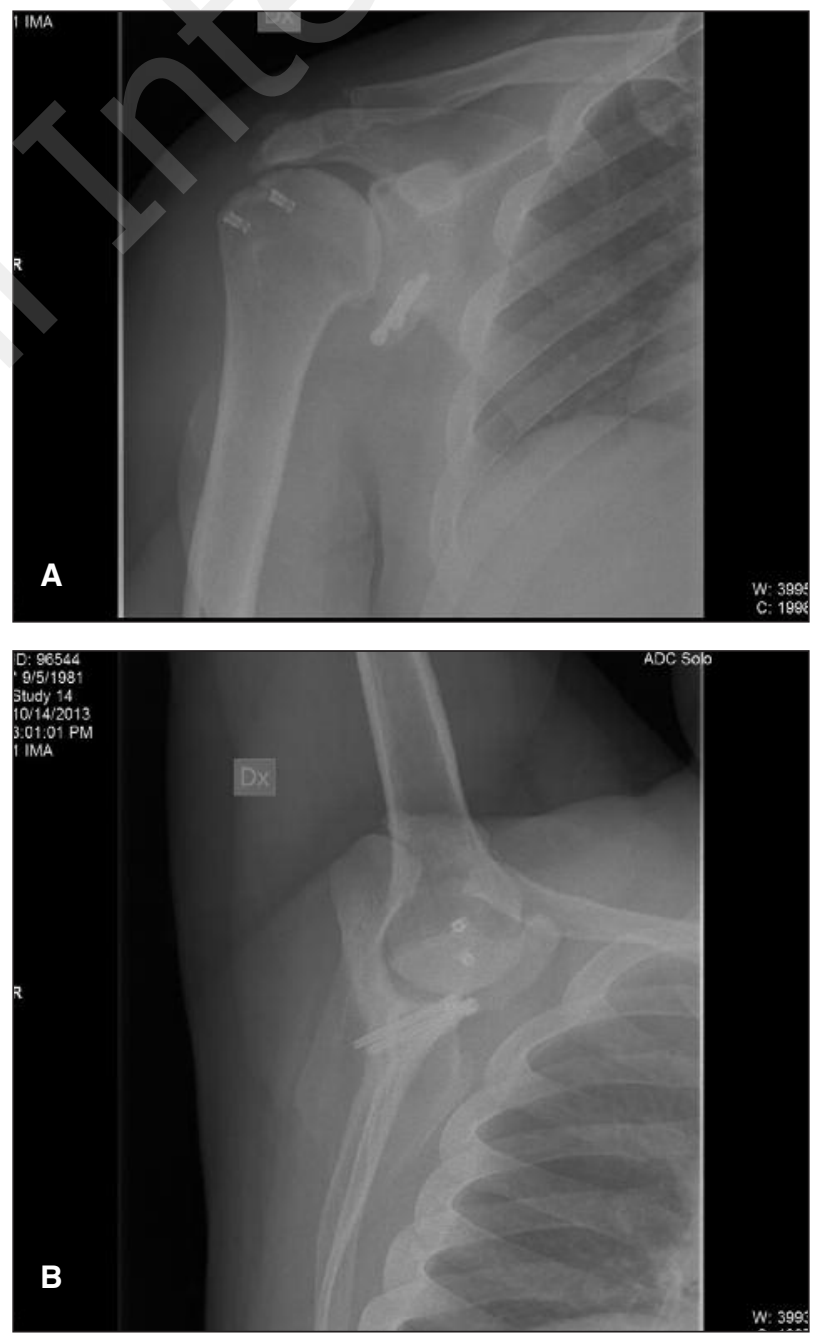

Fig. 1. AP view (A) and Bernageau profile view (B) of the operated shoulder one year post-operatively. 
rotation (ER) bilaterally at $210^{\circ} / \mathrm{sec}$ in $45^{\circ}$ of shoulder abduction. Peak torques were higher in the affected limb during ER (8.5\%) and IR (29.6\%).

\section{Discussion}

Many elderly patients with a shoulder dislocation will also have a tear of the rotator cuff. The current literature reports incidences ranging from 35 to $86 \%$ in patients over 40 years old (3). These data are strikingly different from those recorded in the younger population, where rotator cuff tears rarely occur in conjunction with anterior shoulder dislocation. Taylor and Arciero (4) reported a series of anterior shoulder dislocations in patients under 24 years old from the United States Naval Academy. Of the 63 patients, managed arthroscopically, 97\% had evidence of a Bankart lesion, but no rotator cuff tears were found.

We here presented the case of a young patient with recurrent anterior shoulder dislocation with glenoid bone loss and concurrent irreparable rotator cuff tear. The unusual nature of this case derives from the patient's young age and the extent of his rotator cuff tear. Given the size of the rotator cuff tear and the nature of the combined instability injuries (glenoid bone loss and engaging Hill-Sachs defect), extensive open treatment would have been necessary in order to repair both lesions, therefore we instead elected to perform an arthroscopic bone graft and an LD transfer to address the glenoid bone loss and the cuff tear, respectively, in the hope of minimizing the tissue damage and maximizing the patient's chance of regaining full motion and stability.

In a case such as this, if an arthroscopic Bankart repair is planned, the technical limitations of the procedure clearly need to be considered: the first of these was the large glenoid bone defect. It is also necessary to seriously consider utilizing a bony procedure, rather than a standard capsulolabral Bankart repair. The
Latarjet procedure, transferring the tip of the coracoid process with the attached conjoined tendon, undermines the integrity of the coracoacromial arch. Wellman et al. (5) demonstrated that coracoacromial ligament resection induces an increased glenohumeral translation under passive loading conditions without muscular stabilization. These results underline the importance of preserving the coracoacromial arch and convinced us to use a bone graft.

In conclusion, even though the results in this case were excellent, no treatment recommendations can be made on the basis of this single case. But it can be hypothesized that in a young patient with recurrent anterior shoulder dislocation and rotator cuff deficiency, anterior glenoid bone grafting, preserving the coracoacromial arch, is the key to long-term functional improvement, not only for pain relief but also for obtaining stabilization. Finally, it is to be noted that the time needed for healing of the LD tendon transfer determined the long time (12 months) to full recovery and return to normal activity in this patient.

\section{References}

1. Petriccioli D, Bertone C, Marchi G, Garofalo R. Arthroscopically assisted latissimus dorsi transfer with a minimally invasive harvesting technique: surgical technique and anatomic study. Musculoskelet Surg 2012; 96 Suppl 1:S3540.

2. Petriccioli D, Bertone C, Marchi G. Treating recurrent anterior glenohumeral instability using an autogenous tricortical iliac-crest bone graft: Eden-Hybbinette procedure. In: Di Giacomo G, Costantini A, De Vita A (Eds), Shoulder Instability: Alternative Surgical Techniques. Springer, Milan 2011; 148-177.

3. Porcellini G, Paladini P, Campi F, Paganelli M. Shoulder instability and related rotator cuff tears: arthroscopic findings and treatment in patients aged 40 to 60 years. Arthroscopy 2006; 22:270-276.

4. Taylor DC, Arciero RA. Pathologic changes associated with shoulder dislocations. Arthroscopic and physical examination findings in first-time, traumatic anterior dislocations. Am J Sports Med 1997; 25:306-311.

5. Wellmann M, Petersen W, Zantop T, Schanz S, Raschke MJ, Hurschler C. Effect of coracoacromial ligament resection on glenohumeral stability under active muscle loading in an in vitro model. Arthroscopy2008; 24:1258-1264. 